\title{
Regulation of Glycogen Synthase Kinase $3 \beta$ Functions by Modification of the Small Ubiquitin-Like Modifier
}

\author{
Eun Jeoung Lee ${ }^{1}$, Sung Hee Hyun ${ }^{2}$, Jaesun Chun ${ }^{3}$, Sung Hwa Shin ${ }^{1}$, Kwang Hum Yeon ${ }^{3}$, \\ Min Kyoung Kwak ${ }^{1}$, Tae Yoon Park ${ }^{4}$ and Sang Sun Kang*,1
}

\author{
${ }^{I}$ School of Science Education, Chungbuk National University, Gaeshin-dong, Heungdok-gu, Cheongju, Chungbuk, 361 - \\ 763, Republic of Korea. \\ ${ }^{2}$ Department of Pre-medicine, Eulji University School of Medicine, Daejeon 301-832, Republic of Korea \\ ${ }^{3}$ Department of Biology Education, Korea National University of Education, Chongwon, Chungbuk 363-791, Republic \\ of Korea \\ ${ }^{4}$ Graduate School of Education, Yonsei University, 134 Shinchon-dong, Seodaemun-gu, Seoul 120-749, Republic of Ko- \\ rea
}

\begin{abstract}
Modification of the Small Ubiquitin-like Modifier (SUMO) (SUMOylation) appears to regulate diverse cellular processes, including nuclear transport, signal transduction, apoptosis, autophagy, cell cycle control, ubiquitin-dependent degradation and gene transcription. Glycogen synthase kinase $3 \beta$ (GSK 3 $\beta$ ) is a serine/threonine kinase that is thought to contribute to a variety of biological events, including embryonic development, metabolism, tumorigenesis, and cell death. GSK $3 \beta$ is a constitutively active kinase that regulates many intracellular signaling pathways by phosphorylating substrates such as $\beta$-catenin. We noticed that the putative SUMOylation sites are localized on $\mathrm{K}^{292}$ residue of ${ }^{291} \mathrm{FKFPQ}{ }^{295}$ in GSK $3 \beta$ based on analysis of the SUMOylation consensus sequence. In this report, we showed that the SUMOylation of GSK $3 \beta$ occurs on its $\mathrm{K}^{292}$ residue, and this modification promotes its nuclear localization in COS- 1 . Additionally, our data showed that the GSK $3 \beta$ SUMO mutant (K292R) decreased its kinase activity and protein stability, affecting cell death. Therefore, our observations at first time suggested that SUMOylation on the $\mathrm{K}^{292}$ residue of GSK $3 \beta$ might be a GSK $3 \beta$ regulation mechanism for its kinase activation, subcellular localization, protein stability, and cell apoptosis.
\end{abstract}

Keyword: GSK 3ß, SUMOylation, cell apoptosis, protein stability, subcellular localization, kinase activation.

\section{INTRODUCTION}

SUMO (Small Ubiquitin-like Modifier) modification (SUMOylation) of proteins, especially of transcriptional regulators and nuclear pore proteins has been described [1]. SUMO is a new covalent modification leading to attachment of SUMO to specific lysine residues of target proteins $[2,3]$. SUMO represents a class of ubiquitin-like proteins conjugated, like ubiquitin, by a set of enzymes to cellular proteins [5]. However, SUMOylation does not promote protein degradation and distinct enzymes are involved in SUMOylation. For SUMOylation of substrate proteins, SAE1/SAE2 heterodimer acts as an E1 enzyme in mammals (Aos1/Uba2 in yeast), and Ubc9 acts as an E2 SUMO-conjugating enzyme [6]. The mammalian PIAS (protein inhibitor of activated STAT) family, Ran Bp (Ran binding protein) 2 and the polycomb PC2 repressor, have recently been shown to function as E3-type SUMO ligases [7-10]. The analysis of many SUMOylation substrates indicates that it occurs at a particular sequence, thus the specificity of SUMO conjugation might be conferred by recognition of this sequence by the

*Address correspondence to these authors at the School of Science Education, Chungbuk National University, Gaeshin-dong, Heungdok-gu, Chongju, Chungbuk, 361-763, Republic of Korea

Department of Biology Education, Korea National University of Education, Chongwon, Chungbuk 363-791, Republic of Korea; Tel; +82 43261 3278; Fax; +82 43271 0526; E- mail; jin95324@cbu.ac.kr thioester-linked Ubc9-SUMO conjugate [11-13]. Lysine residues targeted for SUMOylation are often found within specific sequences, such as its consensus sequence [5, 14].

Glycogen synthase kinase $3 \beta$ (GSK $3 \beta$ ) is a constitutively active serine/threonine kinase that regulates many in tracellular signaling pathways by phosphorylating substrates such as $\beta$-catenin $[15,16]$. The phosphorylation of $\beta$-catenin by GSK $3 \beta$ is facilitated by the scaffold protein axin and is inhibited either by GSK 3-binding protein (GBP), also known as Frat (Frequently rearranged in advanced T-cell lymphomas), or Dishevelled. The phosphorylation of $\beta$ catenin by GSK $3 \beta$, resulting in its ubiquitin-mediated proteolysis, is the axis of the canonical Wnt signaling pathway. Thus, GSK $3 \beta$ is thought to contribute to a variety of biological events, such as embryonic development, metabolism, tumorigenesis, and cell death [17]. The function of GSK $3 \beta$ is also regulated through phosphorylation by other protein kinases including Akt, which is a serine/threonine kinase that is activated by phosphatidylinositol 3-kinase (PI3K) signaling and phosphorylates GSK $3 \beta$ on $\mathrm{S}^{9}$, thereby inactivating it $[18,19]$.

During our investigation of the Akt-GSK $3 \beta$ signal transduction pathway, we noticed the putative SUMOylation site $\left(\mathrm{K}^{292}\right.$ in $\left.{ }^{291} \mathrm{FKFPQ}^{295}\right)$ in GSK $3 \beta$ using consensus motif computer analysis (http://www.abgent.com/doc/sumoplot). This finding led us to evaluate whether GSK $3 \beta$ is SUMOy- 
lated or not. And then, we observed the SUMOylation of GSK $3 \beta$ with in vitro or in vivo SUMOylation assay. Additionally, we identified that the SUMOylation site of GSK $3 \beta$ is the $\mathrm{K}^{292}$ residue using site directed mutagenesis analysis. We also characterized the biological significances of GSK $3 \beta$ SUMOylation using a GSK $3 \beta$ kinase assay, confocal microscopy and FACS analysis. Therefore, in this article, our data suggest that SUMOylation in GSK $3 \beta$ is one of the regulation mechanisms for kinase activity, protein stability, and nuclear localization, as well as affecting cell apoptosis. Even though it is unclear how SUMOylation of GSK $3 \beta$ occurs in the cell, we suggest here that SUMOylation on the $\mathrm{K}^{292}$ residue of GSK $3 \beta$ seems to be a new mechanism for its functional regulation.

\section{MATERIALS AND METHODS}

\section{Cell Culture}

COS-1 was purchased from ATCC (Manassas, VA, USA). Media and supplements were obtained from GIBCO (Grandisland, NY, USA). The cell line was maintained in Dulbecco's Modified Essential Medium (DMEM) containing $10 \%$ heat inactivated (for $30 \mathrm{~min}$ at $56^{\circ} \mathrm{C}$ ) fetal bovine serum (FBS), $100 \mathrm{U}$ potassium penicillin/ml, $100 \mu \mathrm{g}$ streptomy$\mathrm{cin} / \mathrm{ml}, 2 \mathrm{mM}$ glutamine and $20 \mathrm{mM}$ sodium bicarbonate. The cells were incubated at $5 \% \mathrm{CO}_{2}, 95 \%$ humidity and $37^{\circ} \mathrm{C}$ and growth medium changed every 3 days. SUMO fusion protein was obtained from Calbiochem (Grandisland, NY). Wild type human GSK $3 \beta$ was purchased in Ha- or GST-tagged mammalian expression vector (GeneCopoeia Co. CA, USA).

\section{Antibodies}

Monoclonal antibody against the Ha epitope or GST was purchased from Santa Cruz Biotech. Inc. (Santa Cruz, CA, USA). Antibodies against GSK $3 \beta$ or human Tau specific antibody were purchased from Santa Cruz Biotech. Inc. (Santa Cruz, CA, USA). actin antibody was purchased from Cell Signaling Technology, Inc. (Cell Siganling Co. MA, USA). Antibodies against Tau 422 Ser phosphor was purchased from Calbiochem. (La Jolla, CA, Germany). Antibodies against SUMO-1 was purchased from ABGENT ( San Diego, CA, USA).

\section{Site-Directed Mutagenesis of GSK $3 \beta$}

To generate GSK 3 $\beta$, K292R, and K340R (UP; 5' -aac tac aca gaa ttt aGG ttc cct caa att aag gca-3', Down; 5'-aat ttg agg gaa CCt aaa ttc tgt gta gtt tgg gtt-3) and (UP; 5' - cgg gac cca aat gtc aGG cta cca aat ggg cga gac-3', Down 5'- ccc att tgg tag CCt gac att tgg gtc ccg taa ttc-3) from GSK $3 \beta$ were used [20] with a "Chameleon" double-stranded site-directed mutagenesis kit (Stratagene, CA, USA), according to the manufacturer's instructions. Every mutation was confirmed by DNA sequencing.

\section{GSK $3 \beta$ Expression Vector Transfection and Purification}

For mammalian expression, Ha-GSK $3 \beta$ wt or GSK $3 \beta$ SUMO mutant construct were transfected into COS- 1 cells using the lipofectin transfection method (Gibco-BRL Co). Transfected cells $\left(2 \times 10^{7}\right)$ were lysed in RIPA lysis buffer. Anti-Ha monoclonal antibody was incubated with $1000 \mu \mathrm{l}$ of pre-cleaned cell lysate and precipitated with protein A aga- rose beads. The beads were then washed three times with excess cell lysis buffer and the final pellet used for the immuno assay to detect SUMOyaltion. Western blots were performed with anti-SUMO-1 antibody to detect the presence of SUMO [3, 21]. To detect the phosphorylation of GSK $3 \beta$ $\mathrm{T}^{216}$ residue, an anti-216 Tyr phospho $\mathrm{Ab}$ (La Jolla, CA, Germany) was used.

\section{Double Immunofluorescence Microscopy}

COS- 1 cells were plated at a low confluence $(\sim 30 \%)$ on two-well Lab-Tek Permanox slides (Nalgene Nunc International, Naperville, IL) and transiently transfected with $\mathrm{Ha}-$ GSK $3 \beta$ wt or Ha-GSK $3 \beta$ SUMO mutant (K292R) plasmid using the lipofectamine procedure. Cells were starved for $36 \mathrm{~h}$ and subsequently treated with $10 \%$ calf serum for $15 \mathrm{~h}$. At no time did cell confluency exceed $60 \%$. Cells were fixed, permeabilized, and processed for indirect double immunofluorescence microscopy as described previously, with minor modifications. Cells were blocked in normal goat and diluted (1:30) in PBS for 15 min, then incubated with affinity-purified, anti- antibodies at 1:150 dilution in combination with a 1:1000 dilution of murine SUMO-1 monoclonal antibodies (ABGENT, San Diego, CA, USA), anti- GSK $3 \beta S^{9}$ residue, an anti-9 Ser phospho $\mathrm{Ab}$ (purchased from Cell Siganling Co. MA, USA), or Ha monoclonal antibodies (Santa Cruz, CA, USA) at room temperature for 1-2 $\mathrm{h}$ on a rocking platform. Washed slides were then incubated for $1 \mathrm{~h}$ at room temperature with 1:150 dilutions of both anti-rabbit fluorescein isothiocyanate-conjugated secondary antibody (Molecular Probes Inc. OR, USA) and Texas red-conjugated goat anti-rabbit secondary antibody (Molecular Probes Inc. CA, USA). Slides were washed and then mounted with Vectashield mounting medium (Vector Laboratories Inc. CA, USA) and examined using Leica TCS SPII AOBS in The Core Facility of Chungbuk National University [20].

\section{Expression and Purification of Recombinant Proteins}

GST tagged GSK $3 \beta$ wt was purchased from GeneCopia TM and its SUMO mutant (K292R) was cloned with the same primer set used for generation of mammalian SUMO mutants. GST tagged protein was expressed in Escherichia coli BL21 and purified with GST-agarose beads according to the manufacturer's instruction (Amersham Biosciences Co). Purified proteins were used for the SUMOylation assay substrate protein.

\section{In vitro SUMO-1 Conjugation Assay}

SUMO-1 conjugation assay was performed in vitro using the SUMO assay kit purchased from Corgen Inc (Taipei, Taiwan), according to the manufacturer's recommended protocol. One microgram of purified GST-tagged GSK $3 \beta \mathrm{mu}-$ tants were mixed with $250 \mathrm{ng}$ of Ubc9, $125 \mathrm{ng}$ of Aos1/Uba2, with or without $2 \mu \mathrm{g}$ of SUMO-1, then incubated for $2 \mathrm{hr}$ at $30^{\circ} \mathrm{C}$ in the presence of $50 \mathrm{mM}$ Tris $[\mathrm{pH}$ $7.5], 5 \mathrm{mM} \mathrm{MgCl} 2$, with or without (for the negative control) $2 \mathrm{mM}$ ATP in a $20 \mu 1$ volume. Reactions were stopped with SDS-PAGE sample buffer and SUMO conjugates separated by SDS-PAGE and analyzed by western blotting using the mouse monoclonal antibody against SUMO-1 to detect GSK $3 \beta$ [3]. The relative optical density (OD) was measured by image analysis of the dried SDS-PAGE gel using the Fuji 
Image Quant software (Fujifilm, Tokyo, Japan), according to the manufacturer's instructions.

\section{GSK $3 \beta$ Kinase Assay}

Human Tau protein was purchased from Biomol (CA, USA). GSK $3 \beta$ kinase assays were performed for 30 minutes at $30{ }^{\circ} \mathrm{C}$ in a $25-\mu 1$ reaction volume containing $[20 \mathrm{mM}$ HEPES, pH 7.2, $10 \mathrm{mM} \mathrm{MgCl} 2,10 \mathrm{mM} \mathrm{MnCl}_{2}, 1 \mathrm{mM}$ dithiothreitol, $0.2 \mathrm{mM}$ EGTA, and $1 \mu \mathrm{g}$ phosphatidylserine], and $1 \mu \mathrm{g}$ human Tau protein as a GSK $3 \beta$ substrate protein. The phosphorylated Tau protein was detected using a Western blot with Tau $\mathrm{S}^{422}$ phosphor specific antibody, purchased from Calbiochem (La Jolla, CA, Germany).

\section{Protein Stability Experiments}

COS- 1 cells $\left(2.5 \times 10^{5}\right.$ cells per well $)$ in $10 \mathrm{~cm}$ plates were transfected with $1.0 \mu \mathrm{g}$ of expression vector with HaGSK $3 \beta$ wt or SUMO mutant plasmid. The medium was replaced with medium containing $200 \mu \mathrm{g} / \mathrm{ml}$ cycloheximide $36 \mathrm{~h}$ after transfection ( 0 -h time point). Cell lysates were harvested at $0,8,16$, and $24 \mathrm{~h}$ then analyzed by immunoprecipitation and Western blotting using anti-Ha antibodies, and assayed in five time repeats. The relative optical density (OD) was measured by image analysis of the dried SDSPAGE gel with the Fuji Image Quant software (Fujifilm, Tokyo, Japan), according to the manufacturer's instructions.

\section{A Glycogen Synthase Kinase $3 \beta$ (GSK $3 \beta$ ) domain and putative SUMOyltion site}

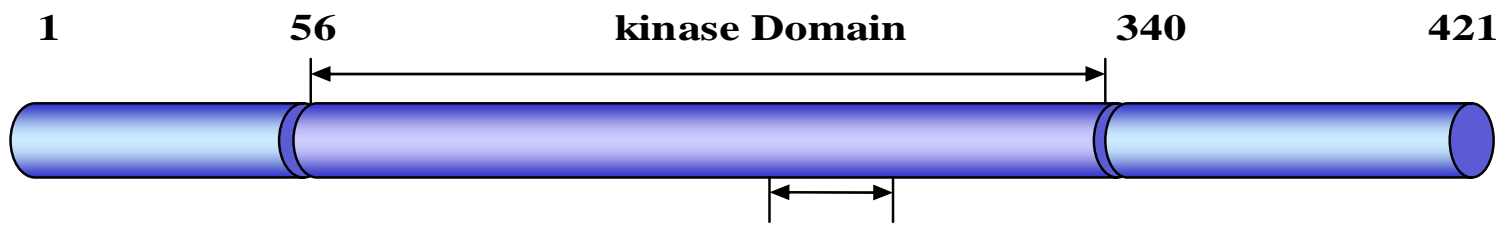

FRAT/Axin binding sites (262-299) 292 residue of 291 FKFPQ295

B
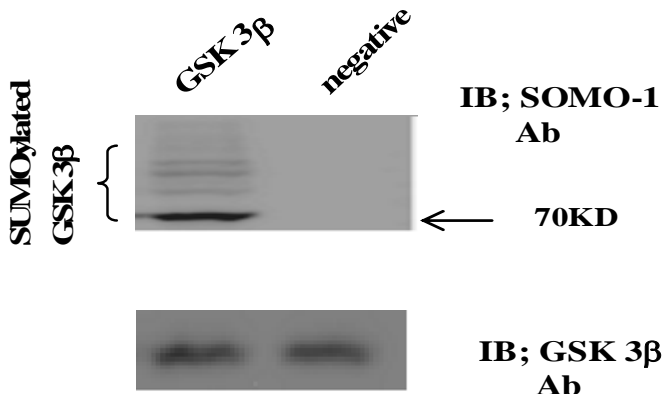

IB ; GSK $3 \boldsymbol{\beta}$

Ab

C

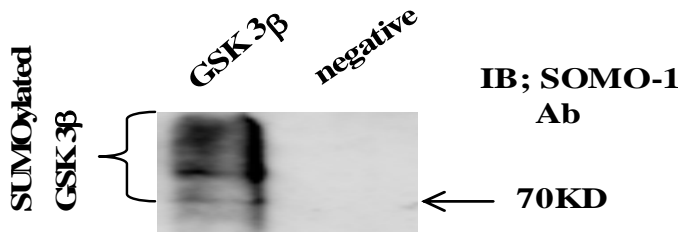

D
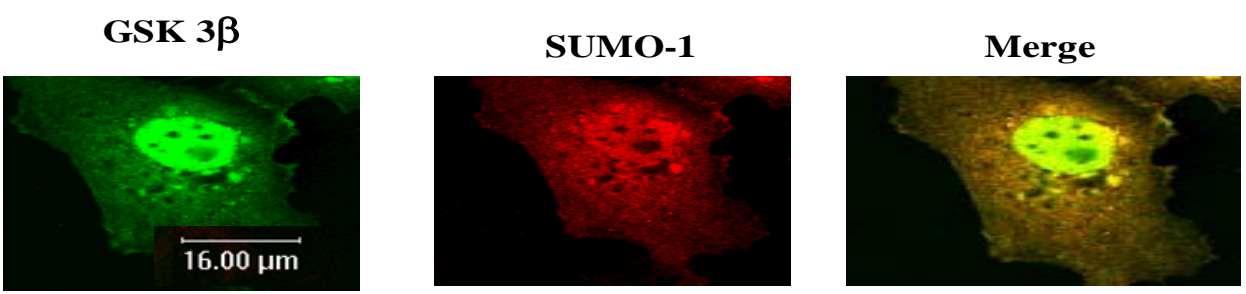

Fig. (1). GSK $3 \beta$ functional domain and SUMOylation.

The Glycogen synthase kinase $3 \beta$ (GSK $3 \beta$ ) functional domains (its protein kinase and FRAT/Axin binding domain) and the putative SUMOylation site $\left(\mathrm{K}^{292}\right.$ in ${ }^{291} \mathrm{FKFPQ}^{295}$ ) is indicated (A). GSK $3 \beta$ SUMO mutant (K292R) was constructed by site directed mutagenesis. GSK $3 \beta$ SUMO mutant (K292R) was inserted into GST fusion (for bacteria) and Ha fusion (for cell line) expression vectors. (B) GSK $3 \beta$ wild type (wt) protein that was purified from E. coli was incubated with a SUMOylation assay kit (See Material and method). For the negative control, the same assay conditions were used without ATP (right lane). A western blot of the same sample was performed with GSK $3 \beta$ monoclonal antibody to monitor the protein amount in the experiment (at bottom). SUMOylated GSK $3 \beta$, as several high molecular weight protein bands, was indicated. (C) A western bolt of the immunopurified GSK $3 \beta$ from COS-1 was performed using the SUMO- 1 specific antibody. SUMOylation of GSK $3 \beta$ was detected as high molecular weight protein bands, as indicated (left lane). For the negative control, an unrelated mouse antibody was used (right lane). To monitor the total protein amount to be used in the cell lysates, the western blot was performed with actin monoclonal antibody (bottom). (D) Confocal microscopic analysis of endogenous GSK $3 \beta$ wt (green color) and SUMO-1 (red color). GSK $3 \beta$ was detected in both the cytoplasm and nuclear region. The SUMO-1 modification proteins were mainly detected in the nuclear region (yellow color). All the figures in this article represent results from three experiments repeated independently. 


\section{FACS Analysis}

Ha-GSK $3 \beta$ (wt), Ha-GSK $3 \beta$ SUMO mutant (K292R), or pcDNA vector was transfected and the rate of apoptosis measured by Annexin V-PE apoptosis detection kit I (BD Biosciences, CA, USA), according to the manufacturer's instructions. Transfected Cells were washed twice in cold PBS and then resuspended in Binding buffer $(0.01 \mathrm{M}$ Hepes/NaOH (pH 7.4) $0.14 \mathrm{M} \mathrm{Nacl}, 2.5 \mathrm{mM} \mathrm{CaCl}_{2}$ ). 1 X10 cells in $100 \mu \mathrm{l}$ were transferred to $5 \mathrm{ml}$ culture tube and adde $5 \mu \mathrm{l}$ of Annexin V-PE and $5 \mu 1$ of 7-Amino-actinomycin D. The cells were vortexed gently and incubated for $15 \mathrm{~min}$ at $25{ }^{\circ} \mathrm{C}$ in the dark. $400 \mu \mathrm{l}$ of binding buffer was added to each tube. Within $1 \mathrm{hr}$, FACS was performed on a Coulter Epics Elite equipped with a gated amplifier and upgraded with enhanced system performance in The Core Facility of Chungbuk National University [20].

\section{RESULTS}

\section{SUMOylation of GSK $3 \beta$ In Vitro and In Vivo}

Using computer analysis of the SUMOylation consensus sequence from GSK $3 \beta$, two sites were found in the Cterminal domain $\left(\mathrm{K}^{292}\right.$ in $\left.{ }^{291} \mathrm{FKFPQ}^{295}\right)$, which is near by the Axin and FRAT binding domains (262-299aa), as shown in Fig. (1A) $[22,23]$. Therefore, we predicted that GSK $3 \beta$ is one of the SUMO modified (SUMOylation) proteins $[3,13]$. To test our prediction, we constructed GSK $3 \beta$ SUMO mutant (K292R) which is indicated in Fig. (1A) below, by site directed mutagenesis. Both the GSK $3 \beta$ wild type (wt) and SUMO mutant (K292R) was inserted into Ha tagged expression vector (for eukaryotic cell) or GST fusion expression vector (for prokaryotic cell).

Initially we performed the in vitro SUMOylation assay using GST-GSK $3 \beta$ fusion protein purified from E. coli to determine whether SUMOylation of GSK $3 \beta$ occurred, as described in the Materials and Methods section [21], using a SUMO assay for GSK $3 \beta$ wt without ATP as a negative control (Fig. 1B, right lane). The western blot of each sample was performed using GSK $3 \beta$ antibody to monitor the protein amount in the experiment (at bottom). As shown in Fig. (1B), SUMOylated GSK $3 \beta$ was detected, including several high molecular weight protein bands (left lane), suggesting that GSK $3 \beta$ is one of SUMOylation proteins in vitro.

To confirm GSK $3 \beta$ SUMOylation in the cell, we performed a western bolt of the immunopurified GSK $3 \beta$ from COS- 1 cells with SUMO- 1 specific antibody, as described in the Materials and Methods section [21]. As shown in Fig. (1C), SUMOylation of GSK $3 \beta$ was detected as high molecular weight protein bands (left lane), similar to the results in Fig. (1B). An unrelated mouse antibody was used as a negative control (right lane). To monitor the total protein amount to be used in the cell lysates, western blot was performed with actin monoclonal antibody (Fig. 1C, bottom). Therefore, the results depicted in Fig. (1C) also suggest that GSK $3 \beta$ was SUMOylated in COS- 1 cells, which is consistent with the results in Fig. (1B).

Confocal microscopy observation of COS-1 cells with GSK $3 \beta$ or SUMO-1 specific antibody was used to observe whether endogenous GSK $3 \beta$ undergoes SUMOylation in the cell. As shown in Fig. (1D), GSK 3 $\beta$, which was detected in both the cytoplasm and the nucleus, was merged with SUMO-1 in the nucleus. These results suggest that endogenous GSK $3 \beta$ in COS- 1 was modified by SUMO- 1 , which is consistent with Fig. (1B and C). Interestingly, SUMOylation of GSK $3 \beta$ seems to be required for its nuclear localization (Fig. 1D). Therefore, overall, these results (Fig. 1A, B, C, and $\mathbf{D})$ suggest that GSK $3 \beta$ is one of the SUMOylation proteins.

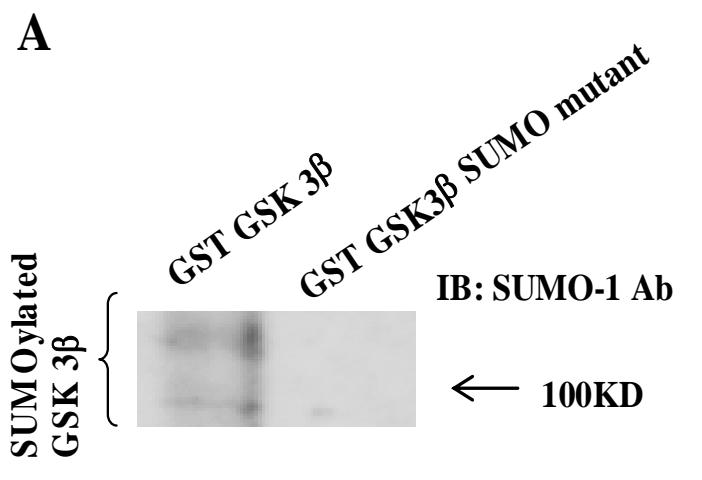

IB: GST Ab

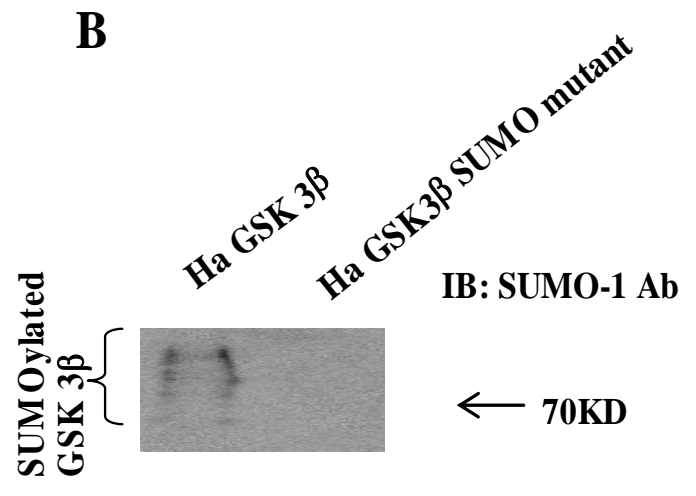

IB: GSK3 $\beta$ Ab

Fig. (2). SUMOylation Site in GSK $3 \beta$.

(A) The purified GST-GSK $3 \beta$ wt or GST-GSK $3 \beta$ SUMO mutant (K292R) fusion protein was used as the substrate protein in the SUMOylation assay as described in the Materials and Methods section. The SUMOylation of GSK $3 \beta$ wt was detected as a high molecular weight protein band (left lane), whereas its SUMO mutant was totally inhibited, as shown (right lane). (B) Ha -GSK $3 \beta$ wt or Ha -GSK $3 \beta$ SUMO mutant was transfected to COS-1 cells and immunoprecipitated with Ha mouse monoclonal antibody. The immunoprecipitants were subjected to the western bolt with SUMO-1, as described in the Materials and Methods section. The SUMOylation of GSK $3 \beta$ wt was indicated as several high molecular weight protein bands (left lane), whereas its SUMO mutant was totally inhibited (right lane). To monitor the GSK $3 \beta$ protein expression, the immunoprecipitants were subjected to the western bolt with GSK $3 \beta$ polyclonal antibody (bottom). 


\section{SUMOylation Sites in GSK $3 \beta$}

As shown in Fig. (1A), we predicted that $\mathrm{K}^{292}$ in $\left({ }^{291} \mathrm{FKFPQ}^{295}\right)$ of GSK $3 \beta$ was the putative SUMOylation sites based on consensus sequence analysis. To test our prediction, we performed the SUMOylation assay in vitro with GSK $3 \beta$ SUMO mutant (K292R). We used GST fusion GSK $3 \beta$ (wt) or GSK $3 \beta$ SUMO mutant, as the substrate protein. Western blotting of the same sample was performed with GST monoclonal antibody to monitor the amount of protein in the experiment (at bottom). We also performed the SUMOylation assay using GST fusion, GSK $3 \beta$ K292R mutant protein, and did not observe the SUMOylation of each mutant protein (data not shown). As shown in Fig. (2A), the SUMOylation of GSK $3 \beta$ SUMO mutant was not detected, whereas that of GST fusion GSK $3 \beta$ (wt) was observed. Therefore, our data suggested that $\mathrm{K}^{292}$ in $\left({ }^{291} \mathrm{FKFPQ}^{295}\right)$ of GSK $3 \beta$ is the putative SUMOylation sites.

To confirm our observation further, we performed a western bolt using the immunopurified Ha-GSK $3 \beta$ wt or $\mathrm{Ha}$ -GSK $3 \beta$ SUMO mutant (K292R) from COS- 1 cells (Fig. 2B), using the SUMO-1 specific antibody. To monitor the total protein amount to be used in the cell lysates, western blot was performed using GSK $3 \beta$ polyclonal antibody (bottom) (Fig. 2B). As shown in Fig. (2B), SUMOylation of GSK $3 \beta$ wt was detected as high molecular weight protein bands (right lane), whereas GSK $3 \beta$ SUMO mutant (K292R) was not. Thus, as indicated in Fig. ( $\mathbf{2 A}$ and $\mathbf{B})$, these data suggest that SUMOylation of GSK $3 \beta$ occurs on $\mathrm{K}^{292}$ residue in ${ }^{291} \mathrm{FKFPQ}^{295}$.

\section{Confocal Microscopic Analysis with GSK $3 \beta$ SUMO Mu- tant}

Next, we indented to determine the biological significance of SUMOylation on $\mathrm{K}^{292}$ of GSK $3 \beta$. Because the confocal microscopy results in Fig. (1D) suggested that SUMOylation of GSK $3 \beta$ occur in the nuclear region, we first determined whether SUMOylation of GSK $3 \beta$ affects its subcellular localization. We performed the confocal microscopic analysis with Ha -GSK $3 \beta$ wt or the SUMO mutant construct, as described in Fig. (1A). The transfected $\mathrm{Ha}-$ GSK $3 \beta$ wt or Ha -GSK $3 \beta$ SUMO mutant (K292R) was detected as green and the SUMO-1 position was detected as red using fluorescence microscopy (Fig. 3). Similar to the results shown in Fig. (1D), SUMO-1 signals were mainly detected in the nucleus (Fig. 3A middle lane). Ha -GSK $3 \beta$ wt was observed in both the cytoplasm and nucleus, similar to the results shown in Fig. (1D). However, the merged between GSK $3 \beta$ with SUMO-1 (yellow color) was detected in the nucleus and not the cytoplasm (Fig. 3A right lane), suggesting that SUMOylation of GSK $3 \beta$ is related to its nuclear subcellular localization. This result also supports the idea
A

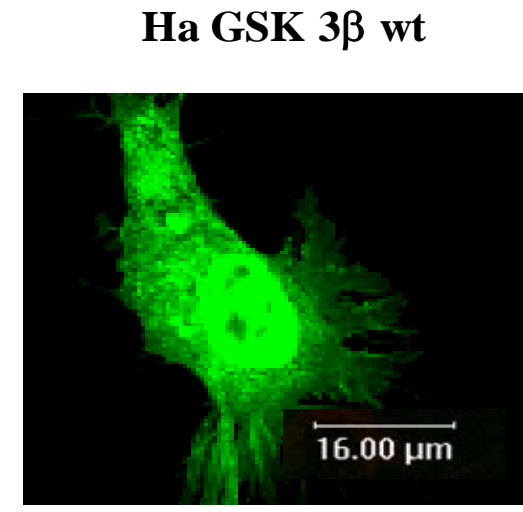

B

Ha GSK $3 \beta$ SUMO mutant

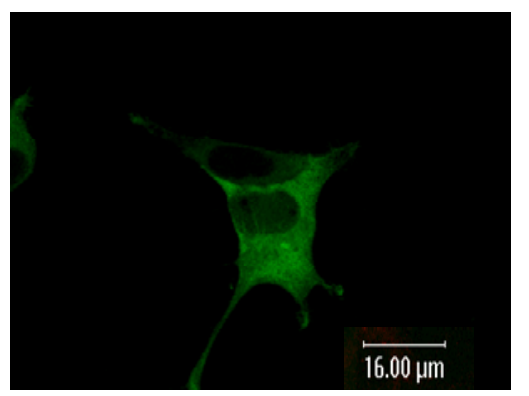

SUMO-1

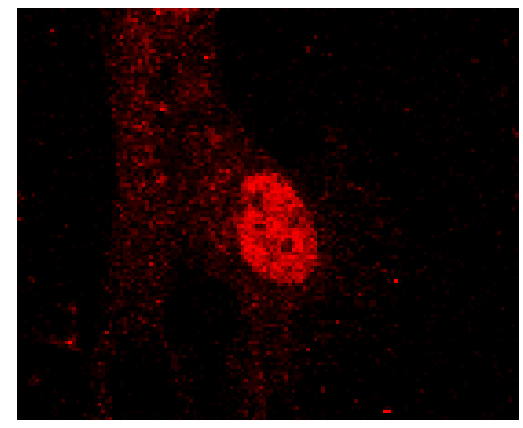

SUMO-1

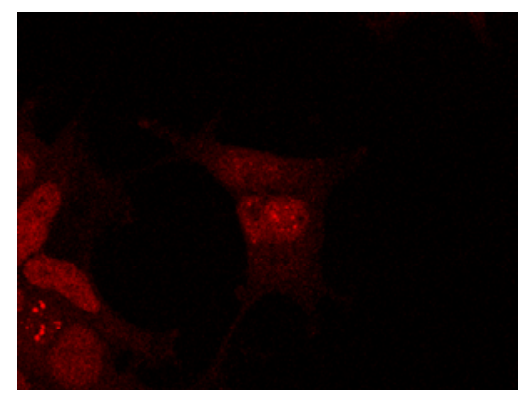

Merge

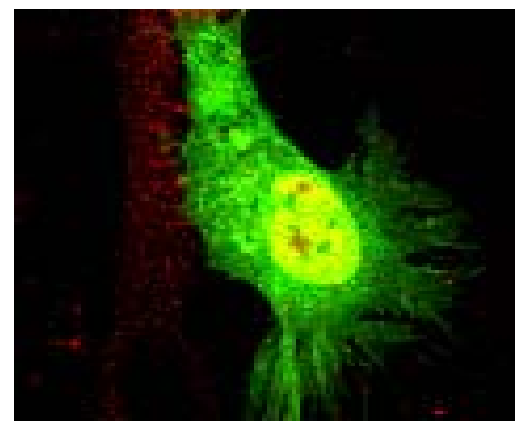

Merge

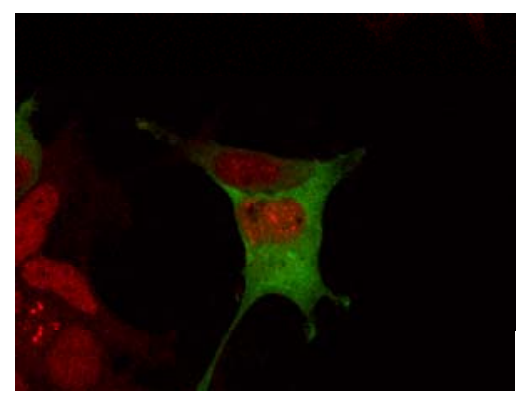

Fig. (3). Confocal microscopic analysis of GSK $3 \beta$ wt or its SUMO mutant

Confocal microscopic analysis of transfected Ha -GSK $3 \beta$ wt (A), Ha -GSK $3 \beta$ SUMO mutant (K292R) (B) was performed to determine whether it merged with SUMO-1 (red color). All Ha -GSK $3 \beta$ constructs were shown as green color. The transfected Ha -GSK $3 \beta$ wt (detected in both the cytoplasm and the nucleus) merged (yellow) with SUMO-1 in the nucleus (A). The transfected Ha -GSK $3 \beta$ SUMO mutant was detected in the cytoplasm, but not in the nucleus (B). The SUMO-1 modification proteins were mainly detected in the nuclear region (B middle lane). GSK $3 \beta$ SUMO mutant in which the SUMOylation site was eliminated was not merged with SUMO-1 in the nucleus (B right lane). 
that GSK $3 \beta$ is one of the SUMOylation proteins, consistent with the results presented in Fig. (1D).

Surprisingly, Ha -GSK $3 \beta$ SUMO mutant was mainly detected in the cytoplasm, not the nucleus, and was not merged (yellow color) with SUMO-1 in the nucleus (Fig. 3B right lane). Therefore, these results suggested that SUMOylation of GSK $3 \beta$ is required for its nuclear localization, consistent with Fig. (1D and 3A). Further, consistent with the results shown in Fig. (2), this result (Fig. 3B) again confirmed that $\mathrm{K}^{292}$ of GSK $3 \beta$ is SUMOylation sites by the confocal microscopic analysis.

\section{SUMOylation of GSK $3 \beta$ is Required for its Kinase Acti- vation}

To further define the biological significance of GSK $3 \beta$ SUMOylation, we compared the kinase activity of GSK $3 \beta$ with that of the GSK $3 \beta$ SUMO mutant. Each Ha-GSK $3 \beta$ wt or its SUMO mutant (K292R) expression vector was transfected into COS-1 cells, and immunoprecipitated with $\mathrm{Ha}$ monoclonal Ab. To monitor the expression of Ha -GSK $3 \beta \mathrm{wt}$ or GSK $3 \beta$ SUMO mutants, western blot was performed with an anti-GSK $3 \beta$ antibody (Fig. 4A). Because it has been reported that 216 tyrosine residue phosphorylation of GSK $3 \beta$ is required for its activation $[17,24]$, we monitored it with its 216 tyrosine phosphorylation specific antibody (Fig. 4B). As shown in Fig. (4B), we observed that 216 tyrosine phosphorylation of the Ha -GSK $3 \beta$ SUMO mutant was reduced compared to that of $\mathrm{Ha}-\mathrm{GSK} 3 \beta$ wt (Fig. 3B), even though the expressed protein amount was almost same (Fig. 4A). Next, we measured the kinase activity with its substrate protein Tau, because Ser 422 of human Tau is phosphorylated by GSK $3 \beta[25,26]$. As shown in Fig. (4C), we observed that the kinase activity of the GSK $3 \beta$ SUMO mutant was reduced to half that of the GSK $3 \beta$ wt, consistent with its 216 tyrosine phosphorylation result (Fig. 3B). Taken together, our data suggest that SUMOylation of GSK $3 \beta$ is also required for not only its 216 tyrosine phosphorylation, but also its kinase activity. To eliminate cell line specificity, we performed the experiment using NIH 3T3 cells and obtained the same results (data not shown).

\section{The Effect of GSK $3 \beta$ SUMOylation on Protein Stability}

To evaluate the effect of SUMOylation on GSK $3 \beta$ protein stability, we performed the pulse-chase experiments as described in the Materials and Methods section. Each Ha GSK $3 \beta$ (wt or SUMO mutant) expression vector was transfected into COS-1 cells and immunoprecipitated with $\mathrm{Ha}$ monoclonal $\mathrm{Ab}$ following cyclohexamide treatment (Fig. 5A). GSK $3 \beta$ proteins were chased for the indicated time periods $(0,8,16,24 \mathrm{hr})$, and then immunoprecipitated with a polyclonal anti- Ha antibody and subjected to SDS-PAGE followed by western blot with GSK $3 \beta$ antibody. To control the protein amount, we monitored the actin in each sample by western blotting (Fig. 5A). The quantification of the pulse-chase experiment, as determined by image analysis of the dried SDS-PAGE gel using the Fuji Image Quant software, is shown in Fig. (5B). As shown in Fig. (5), the protein stability of the GSK $3 \beta$ wt was twice that of the GSK $3 \beta$ SUMO mutant, suggesting that SUMOylation on the $\mathrm{K}^{292}$ of GSK $3 \beta$ seems necessary for protein stability.

\section{IP; Anti-Ha}

A

B IB; Anti-GSK3 $\beta$
$Y^{216}$ phospho Ab

IB; Anti-GSK3 $\beta$

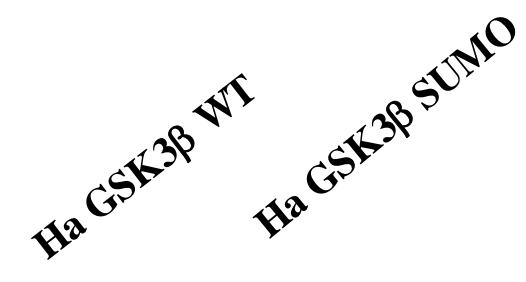

Relative OD

\author{
IB: Anti-Tau \\ $S^{422}$ phospho Ab
}

$1 \quad 0.5$

$1 \quad 0.5$
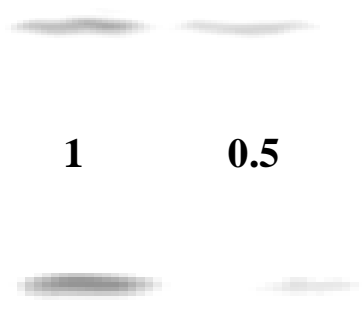

100 30

\section{Relative Activity (Arbitrary unit)}

Fig. (4). Kinase activity of GSK $3 \beta$ wt or its SUMO mutant.

The immunopurified Ha -GSK $3 \beta$ wt or its SUMO mutant (K292R) protein with Ha Ab from COS-1 was immunoblotted with GSK $3 \beta$ (A) or Anti- GSK $3 \beta$ Tyr 216 phospho Ab polyclonal antibody (B). The relative optical density (OD), as determined by image analysis with the Fuji Image Quant software, is indicated below. The GSK $3 \beta$ kinase activity was measured using human Tau protein as a substrate (C). $S^{422}$ residue phosphorylation of human Tau protein was detected with its specific antibody. The relative GSK $3 \beta$ activity by image analysis with the Fuji Image Quant software is indicated below. Results shown are one of five repeated experiments 
$\mathbf{0}$ 4

GSK $3 \beta$ wt

A

Actin

GSK $3 \beta$

SUMO mutant

Actin
8

\section{$16 \mathrm{hr}$ (Chasing time)}

B

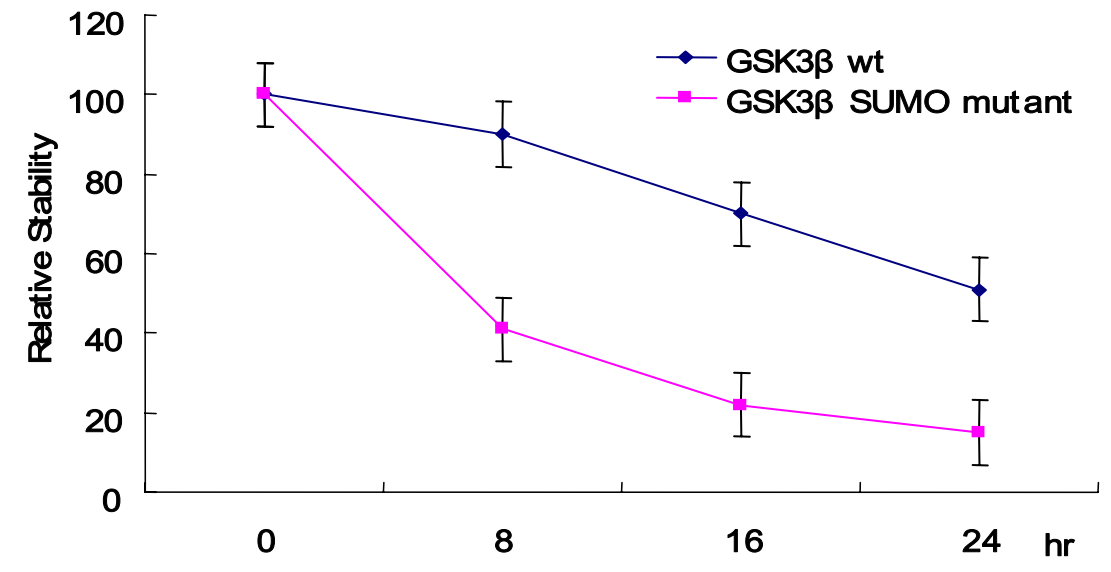

Fig. (5). Protein stability of GSK $3 \beta$ wt and its SUMO mutant.

Ha -GSK $3 \beta$ or GSK $3 \beta$ SUMO mutant (K292R) was transfected into COS-1 cells and the cells treated with cyclohexamide. The GSK $3 \beta$ proteins were chased for the indicated time periods. Ha-GSK $3 \beta$ proteins were immunoprecipitated with a polyclonal anti- Ha antibody and subjected to SDS-PAGE followed by western blotting with a monoclonal GSK $3 \beta$ antibody (A). To monitor the protein amount, an equal amount of cell lysate was subjected to western blotting with an actin antibody. Results shown are one of five repeated experiments. Quantification of the pulse-chase experiment is shown in (B) by image analysis with the Fuji Image Quant software.

\section{The Effect of GSK $3 \beta$ SUMOylation on Cell Viability}

We measured cell viability using FACS analysis to determine whether SUMOylation on $\mathrm{K}^{292}$ of GSK $3 \beta$ influenced cell viability. As shown in Table 1, the FACS results indicate that the GSK $3 \beta$ SUMO mutant (K292R) increased the cell survival rate significantly compared to the Ha -GSK $3 \beta$ wt or pcDNA vector alone. Thus, GSK $3 \beta$ SUMO mutant was only less effective on apoptosis than the Ha -GSK $3 \beta$ wt or pcDNA vector alone (Table 1).

Table 1. Cell viability of GSK $3 \beta$ wt and its SUMO mutant.

\begin{tabular}{|c|c|}
\hline GSK 3 $\beta$ construct & Rate of apoptosis $(\%)$ Be FACS \\
\hline \hline Ha-GSK3 $\beta(\mathrm{wt})$ & $25+/-3$ \\
\hline Ha-GSK3 $\beta$ (SUMO mutant) & $5+/-2$ \\
\hline pcDNA (vector only) & $10+/-2$ \\
\hline
\end{tabular}

Ha -GSK $3 \beta$ (wt) or (SUMO mutant, K292R) or pcDNA vector was transfected and the rate of apoptosis measured by FACS. Ha -GSK $3 \beta$ SUMO mutant (K292R), which was dominantly localized in the cytoplasm, promoted cell survival (but not cell apotosis) when compared to GSK $3 \beta$ wt constructs. Results shown are the average of five repeated experiments. For details, see the Materials and Methods section.
In summary, our results indicate that SUMOylation on $\mathrm{K}^{292}$ of GSK $3 \beta$ regulates protein stability, kinase activity, nuclear localization, and cell apoptosis. Therefore, our observations suggest that SUMOylation is a GSK $3 \beta$ functional modification for its regulation.

\section{DISCUSSION}

Because GSK $3 \beta$ is a multi-functional protein kinase that may regulate biological functions, such as embryonic development, metabolism, tumorigenesis, and cell death, by regulation of many intracellular signaling pathways through phosphorylation substrates, such as $\beta$-catenin, the characterization of GSK $3 \beta$ modification is essential to understand its function and regulation [15-17].

To understand the modification of GSK $3 \beta$, we first tested whether GSK $3 \beta$ is a SUMOylated protein or not. After computer analysis of the SUMOylation motif indicated that GSK $3 \beta$ seems to be SUMOyalted, we confirmed that GSK $3 \beta$ is one of the SUMOylated proteins by conducting a SUMOylation assay, and through confocal microscopy analysis (Fig. 1D and 3). After confirming that GSK $3 \beta$ is a SUMOylated protein, we intended to know which lysine residues of GSK $3 \beta$ are SUMOylated. To do so, we per- 
formed site directed mutagenesis using computer SUMOyaltion consensus motif analysis (Fig. 1A) and found that SUMOylation occurs on the $\mathrm{K}^{292}$ in ${ }^{291} \mathrm{FKFPQ}^{295}$ of GSK $3 \beta$ (Fig. 2 and 3). Next, to identify the biological significance of GSK $3 \beta$ SUMOylation, we compared its protein kinase activity, subcellular localization, and stability with those of the GSK $3 \beta$ SUMO mutant and found that SUMOylation of GSK $3 \beta$ affected its biological roles, including protein kinase activity, subcellular localization, stability, and cell viability. (Fig. 3-5, Table 1). Therefore, although the molecular mechanism underlying GSK $3 \beta$ SUMOylation and its regulation remain unknown, our data suggest that SUMOylation occurs on GSK $3 \beta$ protein as a novel posttranslational step for GSK $3 \beta$ functional regulation. Further studies are essential to elucidate the molecular mechanism involved in GSK $3 \beta$ mediated signal transductions through SUMOylation. In particular, it will be important to determine exactly when and where SUMOylation of GSK $3 \beta$ occurs, as well as how it is regulated, because GSK $3 \beta$ is involved in a variety of human diseases, including cancer and Alzheimer's [22, 23, 27-29].

It has been reported that SUMO-1 (but not SUMO-2, 3 and 4) monomerically conjugates the $\varepsilon$ amino group of the $K$ residue in SUMO-1 acceptor consensus sequences [1, 2, 12, 30]. As shown in Fig. (1B and C), however, the SUMOylation of GSK $3 \beta$ wt was detected as several high molecular weight protein bands $(\sim 200 \mathrm{kD})$, which indicates that multiple SUMOylation on several other $\mathrm{K}$ residues of GSK $3 \beta$ occurred. Even though SUMO forms a homo or hetero dimmer in the cell, SUMOyaltion by other SUMO isotypes (SUMO-2, 3 and 4) may be excluded in this experiment, because SUMO-1 monoclonal antibody was used. We noticed several $\mathrm{K}$ residues in GSK $3 \beta$ where multiple SUMOylation was possible on GSK $3 \beta$ by SUMO-1 [31]. However, it is presently unclear why SUMOylation of GSK $3 \beta$ occurs as high molecular weight protein bands in vivo and in vitro in SUMOylation assays conducted using SUMO-1 specific antibody.

Our identification of and $\mathrm{K}^{292}$ residue of GSK $3 \beta$ as targets for the majority of SUMOylation will help determine whether it plays a role in the normal $\beta$ catenin degradation assembly. Phosphorylation of $\beta$-catenin by GSK $3 \beta$ has been shown to be facilitated by the scaffold protein axin and is inhibited either by GSK3-binding protein (GBP), also known as Frat (Frequently rearranged in advanced T-cell lymphomas), or by Dishevelled [22, 23, 27-29]. Thus, basing on our results, which indicate that the GSK $3 \beta$ SUMO mutant promotes cell survival (Table 1), it seems that the GSK $3 \beta$ SUMO mutant facilitates its dissociation with axin but facilitates association with GBP/Frat or Dishevelled (thus staying in the cytoplasm), resulting in inhibition of phosphorylation of $\beta$-catenin and its ubiquitin-mediated proteolysis. SUMOylation on the $\mathrm{K}^{292}$ residue of GSK $3 \beta$ seems to regulate not only protein interaction with Frat/Axin, but also its activation. SUMOylation on the $\mathrm{K}^{292}$ residue of GSK $3 \beta$ also seems to be one of the cell death signals (Table 1), resulting from the increase of its protein stability and nuclear localization (Fig. 1D, Fig. 3). Therefore, we speculated that because GSK $3 \beta$ is one of the major effectors of Wnt signaling $[15,16,17]$, SUMOylation on the $\mathrm{K}^{292}$ residue of GSK $3 \beta$ affected cell survival (Table 1). Even though it is unknown how SUMOylation of GSK $3 \beta$ regulates phosphorylation of its substrates, such as $\beta$-catenin, we are now evalu- ating the effect of GSK $3 \beta$ SUMOylation on phosphorylation of $\beta$-catenin, its stability and Wnt signaling.

It is presently unknown how GSK $3 \beta$ is transported in the nuclear region, where SUMOylation occurs. Since GSK $3 \beta$ is known as a cytoplasmic and nuclear protein [32, 33], GSK $3 \beta$ seems to have its own nuclear localization sequence (NLS) or nuclear export sequence (NES). We did not notice any NLS or NES consensus sequence homology around the SUMOylation site, $\mathrm{K}^{292}$ [34-36]. However, we can not rule out the possibility that our GSK $3 \beta$ SUMO mutant (K292R) influences its own NLS or NES function directly or indirectly, because of the diversity of the NLS or NES consensus sequence $[34,35]$.

Even though it has been reported that accumulation of GSK $3 \beta$ in the nucleus is related to the cell cycle, we do not presently know whether our results regarding GSK $3 \beta$ nuclear localization and its SUMOylation is related to cell cycle regulation $[32,33]$. It has been demonstrated that Akt, which is activated by phosphatidylinositol 3-kinase (PI3K) signaling, phosphorylates GSK $3 \beta$ on $S^{9}$, thereby inactivating it $[18,19]$. Our preliminary data suggested that phosphorylation of GSK $3 \beta$ on $S^{9}$ seems to be unrelated to SUMOylation (data not shown), however it is still unknown what types of cell signals stimulate SUMOylation of GSK $3 \beta$ on $\mathrm{K}^{292}$. Even though we performed the experiment using NIH 3T3 cells and observed the same results as described above, due to the possibility of cell line specificity, we can not say for certain that SUMOylation of GSK $3 \beta$ on $\mathrm{K}^{292}$ regulates its kinase activity, subcellular localization, protein stability, and apoptosis in normal cells. Further, we can not rule out the possibility that the mutation on $\mathrm{K}^{292}$ of GSK $3 \beta$ affect directely on its protein kinase activity, regardless of its SUMOylation.

E1 enzyme, E2 SUMO-conjugating enzyme, and E3 SUMO ligases involved in GSK $3 \beta$ SUMOylation still remain to be characterized and there mechanism identified. Therefore, more studies will be required to elucidate the physiological significance of SUMOylation on $\mathrm{K}^{292}$ residue of GSK $3 \beta$. Because lysine serves as the attachment site for several modifications, including ubiquitination, acetylating, and methylamine [37], it seems important that multiple SUMOylation on other lysine residues of GSK $3 \beta$, which is trigged by SUMOylation on the $\mathrm{K}^{292}$ residue, also plays a role by antagonizing other post-translational modifications. Because the half-life of the GSK $3 \beta$ SUMO mutant was reduced compared to that of the GSK $3 \beta$ wt (Fig. 5), we speculated that the GSK $3 \beta$ SUMO mutant is more ubiqutinized than that of the GSK $3 \beta w t$. However, the relationship between SUMOyaltion and ubiquitinization (or other modification) of GSK $3 \beta$ also remains to be characterized.

Currently, four SUMO subtypes (SUMO-1, 2, 3 and 4) have been identified $[5,14,38]$. The SUMOylation consensus sequences for each subtype are known to be the same as SUMO-1 ( $\Phi \mathrm{KxE} / \mathrm{D}$, where $\Phi$ represents $\mathrm{L}, \mathrm{I}, \mathrm{V}$ or $\mathrm{F}$ and $\mathrm{x}$ is any amino acid) [11-13]. However, the $K^{292}$ residue in ${ }^{291}$ FKFPQ $^{295}$ of GSK $3 \beta$ does not belong to the exact SUMOylation consensus sequences $(\Phi \mathrm{K} \times \mathrm{E} / \mathrm{D}$, where $\Phi$ represents $\mathrm{L}, \mathrm{I}, \mathrm{V}$ or $\mathrm{F}$ and $\mathrm{x}$ is any amino acid). We do not presently know how great an effect the difference between the GSK $3 \beta$ amino acid sequence and the consensus sequences has on SUMOylation. Therefore, the SUMO subtypes that modify GSK $3 \beta$ should be characterized, and the SUMOyla- 
tion subtype that controls each specific GSK $3 \beta$ function should be determined.

In conclusion, point mutagenesis analysis suggested that SUMOylation of GSK $3 \beta$ occurs on its $\mathrm{K}^{292}$ residue, which is overlapped with the Frat or Axin binding domain by SUMO1 (Fig. 1A), and SUMOylation of GSK $3 \beta$ promotes its nuclear localization and protein stability, resulting in the stimulation of cell apoptosis. Overall, our results suggest that SUMOylation on the $\mathrm{K}^{292}$ residue of GSK $3 \beta$ may be a new GSK $3 \beta$ regulation mechanism for kinase activity, subcellular localization, protein stability, and apoptosis. Our findings may also provide a new intervention clue to cure the many human diseases caused by the abnormal regulation of GSK $3 \beta$ mediated signal transductions, such as cancers, diabetes, and Alzheimer's disease.

\section{ACKNOWLEDGMENT}

This work was supported by KOSEF Grant (R01-2006000-10167-0) to SSK. We appreciated The Core Facility of Chungbuk National University.

\section{REFERENCES}

[1] Gill, G. SUMO and ubiquitin in the nucleus: different functions, similar mechanisms? Genes Dev., 2004, 18, 2046-2059.

[2] Wilson, VG.; Rangasamy, D. Intracellular targeting of proteins by sumoylation. Exp. Cell Res., 2001, 271, 57-65.

[3] Dorval, V.; Fraser, P.E. Small ubiquitin-like modifier (SUMO) modification of natively unfolded proteins Tau and alphasynuclein. J. Biol. Chem., 2006, 281, 9919-9924

[4] Kikuchi, A.; Kishida, S.; Yamamoto, H. Regulation of Wnt signaling by protein-protein interaction and post-translational modifications. Exp. Mol. Med., 2006, 38, 1-10.

[5] Melchior, F.; Hengst L. SUMO-1 and p53. Cell Cycle, 2002, 1, 245-249.

[6] Boggio, R.; Colombo, R.; Hay, R.T.; Draetta, G.F.; Chiocca, S. A mechanism for inhibiting the SUMO pathway. Mol. Cell, 2004, 16, 549-561.

[7] Pichler, A.; Gast, A.; Seeler, J.S.; Dejean, A.; Melchior, F. The nucleoporin RanBP2 has SUMO1 E3 ligase activity. Cell, 2002, 108, 109-120.

[8] Kagey, M.H.; Melhuish, T.A.; Wotton, D. The polycomb protein Pc2 is a SUMO E3. Cell, 2003, 113: 127-137.

[9] Bischof, O.; Schwamborn, K.; Martin, N.; Werner, A.; Sustmann, C.; Grosschedl, R.; Dejean, A. The E3 SUMO ligase PIASy is a regulator of cellular senescence and apoptosis. Mol. Cell, 2006, 22, 783-794.

[10] van Waardenburg, R.C.; Duda, D.; M.; Lancaster, C.S.; Schulman, B.A.; Bjornsti, M.A. Distinct functional domains of Ubc9 dictate cell survival and resistance to genotoxic stress. Mol. Cell Biol., 2006, 26, 4958-4969.

[11] Duprez, E.; Saurin, A.J.; Desterro, J.M.; Lallemand-Breitenbach, V.; Howe, K.; Boddy, M.N.; Solomon, E.; de The, H.; Hay, R.T.; Freemont, P.S. SUMO-1 modification of the acute promyelocytic leukemia protein PML: implications for nuclear localization. J. Cell Sci., 1999, 112, 381-393.

[12] Sampson, D.A.; Wang, M.; Matunis, M.J. The small ubiquitin-like modifier-1 (SUMO-1) consensus sequence mediates Ubc9 binding and is essential for SUMO-1 modification. J. Biol. Chem., 2001, 276, 21664-21669.

[13] Yang, S.H.; Galanis, A.; Witty, J.; Sharrocks, A.D. An extended consensus motif enhances the specificity of substrate modification by SUMO. EMBO J., 2006, 25, 5083-5093.

[14] Zhang, H.; Saitoh, H.; Matunis, M.J. Enzymes of the SUMO modification pathway localize to filaments of the nuclear pore complex. Mol. Cell Biol., 2002, 22, 6498-6508.

[15] Cadigan, K.M.; Liu, YI. Wnt signaling: complexity at the surface. J. Cell. Sci., 2006, 119, 395-402.

[16] Kikuchi, A.; Yamamoto, H.; Kishida, S. Multiplicity of the interactions of Wnt proteins and their receptors. Cell Signalling, 2007, 19, 659-671.
[17] Doble, B.W.; Woodgett, J.R. GSK-3: tricks of the trade for a multitasking kinase. J. Cell Sci., 2003, 116, 1175-1186.

[18] Moule, S.K.; Welsh, G..I.; Edgell, N.J.; Foulstone, E.J.; Proud, C.G..; Denton, R.M. Regulation of protein kinase B and glycogen synthase kinase- 3 by insulin and beta-adrenergic agonists in rat epididymal fat cells. Activation of protein kinase B by wortmanninsensitive and -insensitive mechanisms. J. Biol. Chem., 1997, 272, 7713-7719.

[19] Delcommenne, M.; Tan, C.; Gray, V.; Rue, L.; Woodgett, J.; Dedhar, S. Phosphoinositide-3-OH kinase-dependent regulation of glycogen synthase kinase 3 and protein kinase B/AKT by the integrin-linked kinase. Proc. Natl. Acad. Sci. USA, 1998, 95, 1121111216.

[20] Chun, J.; Kwon, T.; Lee, E.J.; Kim, C.H.; Han, Y.S.; Hong, S.K.; Hyun, S.; Kang, S.S. 14-3-3 Protein mediates phosphorylation of microtubule-associated protein GSK3 b by serum- and glucocorticoid-induced protein kinase 1. Mol. Cells, 2004, 18, 360-368.

[21] Lee, E.J.; Hyun, S.H.; Chun, J.; Ahn, H R.; Kang, S.S. Facilitation of SUMO (Small Ubiquitin-like Modifier) Modification at Tau 340-Lys Residue (a Microtubule- associated protein) through phosphorylation at 214-Ser residue. Int. Biosci., 2007, 11, 39-50.

[22] Thomas GM, Frame S, Goedert M, Nathke I, Polakis P, Cohen PA. GSK3-binding peptide from FRAT1 selectively inhibits the GSK3catalysed phosphorylation of axin and beta-catenin. FEBS Lett., 1999, 458, 247-251.

[23] Farr GH, 3rd Ferkey DM, Yost C, Pierce SB, Weaver C, Kimelman D. Interaction among GSK-3, GBP, axin, and APC in Xenopus axis specification. J Cell Biol., 2000, 148, 691-702.

[24] Hughes K, Nikolakaki E, Plyte SE, Totty EF, Woodgett JR. Modulation of glycogen synthase kinase-3 family by tyrosine phosphorylation. EMBO J., 1993, 12, 803-808.

[25] Hanger, D.P.; Hughes, K.; Woodgett, J.R.; Brion, J.P.; Anderso, R.H.; Reith, A.D. Gycogen synthase kinase-3 induces Alzehimer's disease-like phosphorylation of tau: generation of paired helical filament epitopes and neuronal localization of the kinase. Neuro. Sci. Lett., 1992, 147, 58-62.

[26] Sengupta, A.; Novak, M.; Grundke-Iqbal, I.; Iqbal, K. Regulation of phosphorylation of tau by cyclin-dependent kinase 5 and glycogen synthase kinase-3 at substrate level. FEBS Lett., 2006, 580, 5925-5933.

[27] Dajani, R.; Fraser, E.; Roe, S.M.; Yeo, M.; Good, V.M.; Thompson,V.; Dale, T.C.; Pearl, L.H. Structural basis for recruitment of glycogen synthase kinase 3 beta to the axin-APC scaffold complex. EMBO J., 2003, 22, 494-501.

[28] Chou, H.Y; Howng, S.L.; Cheng, T.S.; Hsiao, Y.L.; Lieu, A.S.; Loh, J.K.; Hwang, S.L.; Lin, C.C.; Hsu, C.M.; Wang, C.; Lee, C.I.; Lu, P.J.; Chou, C.K.; Huang, C.Y.; Hong, YR. GSKIP is homologous to the Axin GSK3beta interaction domain and functions as a negative regulator of GSK3beta. Biochemistry, 2006, 45, 1137911389.

[29] Fujii, N.; You, L.; Xu, Z.; Uematsu, K.; Shan, J.; He, B.; Mikami, I.; Edmondson, L.R.; Neale, G.; Zheng, J, Guy, R.K., Jablons, D.M. An antagonist of dishevelled protein-protein interaction suppresses beta-catenin-dependent tumor cell growth. Cancer Res., 2007, 67: 573-579.

[30] Hietakangas, V.; Anckar, J.; Blomster, H.A.; Fujimoto, M.; Palvimo, J.J.; Nakai, A.; Sistonen, L. PDSM, a motif for phosphorylation-dependent SUMO modification. Proc Nat l. Acad. Sci. USA, 2006, 103, 45-50.

[31] Friedhoff, P.; von Bergen, M.; Mandelkow, E.M.; Mandelkow, E. Structure of GSK3 b protein and assembly into paired helical filaments. Biochim. Biophys. Acta, 2000, 1502, 122-132.

[32] Diehl, J.A.; Cheng, M.; Roussel, M.F.; Sherr, C.J. Glycogen synthase kinase-3beta regulates cyclin D1 proteolysis and subcellular localization. Genes Dev., 1998, 12, 2929-3511.

[33] Litovchick, L.; Chestukhin, A.; DeCaprio, J.A. Glycogen synthase kinase 3 phosphorylates RBL2/p130 during quiescence. Mol. Cell Biol., 2004, 24, 8970-8980.

[34] Moroianu, J. Nuclear import and export pathways. J. Cell Biochem., 1999, 33, 76-83.

[35] Schwoebel, E.D.; Moore, M.S. The control of gene expression by regulated nuclear transport. Essays Biochem., 2000, 36, 105-113.

[36] Lee, B.J.; Cansizoglu, A.E.; Suel, K.E.; Louis, T.H.; Zhang, Z.; Chook, Y.M. Rules for nuclear localization sequence recognition by karyopherin beta 2. Cell, 2006, 126, 543-558. 
[37] Feinstein, S.C.; Wilson, L. Inability of GSK3 b to properly regulate neuronal microtubule dynamics: a loss-of-function mechanism by which GSK3 b might mediate neuronal cell death. Biochem. Biophys. Acta, 2005, 173, 268-279.
[38] Bohren, K.M.; Nadkarni, V.; Song, J.H.; Gabbay, K.H.; Owerbach, D. A M55V polymorphism in a novel SUMO gene (SUMO-4) differentially activates heat shock transcription factors and is associated with susceptibility to type I diabetes mellitus. J. Biol. Chem., 2004, 279, 27233-27238.

(C) Lee et al.; Licensee Bentham Open.

This is an open access article distributed under the terms of the Creative Commons Attribution License (http://creativecommons.org/licenses/by/2.5/), which permits unrestrictive use, distribution, and reproduction in any medium, provided the original work is properly cited. 